\title{
Intelligent Drip Irrigation and Animals Entrance Avoiding System Using Wireless Sensor Network with Renewable Sources
}

\author{
M.Dananandhini, R.Murugasami \\ Nandha Engineering College
}

\begin{abstract}
Agriculture uses 85\% of available freshwater resources worldwide, and this percentage will continue to be dominant in water consumption because of population growth and increased food demand.. There are many systems to attain water savings in various crops, from basic ones to more technologically advanced ones. In existing system, plant water status was monitored and irrigation scheduled based on canopy temperature distribution of the plant, which was acquired with thermal imaging. In addition, other systems have been developed to schedule irrigation of crops and optimize water use by means of a crop water stress index they main problem in this due to fuzzy logic optimization mathematical calculation is to become difficult and it is not having animal entry avoidance.To overcome the mentioned problems, system is atomized using soil moisture, temperature ,humidity and animal entrance to the farm can be predicted to through PIR and sound sensor they sensors parameters send through WSN and monitoring system is provided on the internet web page. Notification also given to the mobile phone. This system is fully efficient automation for irrigation through photovoltaic cells. The simulation of this project will be carried out by using MPLAB IDE andPROTEUS. To verify the functionality and monitor the soil, temperature humidity and PIR sensor levels PROTEUS tool is used. The system design is implemented in Embedded C language.
\end{abstract}

\section{INTRODUCTION}

Agriculture uses $85 \%$ of available freshwater reso-urces worldwide, and this percentage will continue to bedominant in water consumption because of population growth and increased food demand. There is an urgent need to create strategies based on science and technology for sustainable use of water, including technical, agronomic, managerial, and institutional improvements .

There are many systems to achieve water savings in various crops, from basic ones to more technologically advanced ones. For instance, in one system plant water status was monitored and irrigation scheduled based on canopy temperature distribu-tion of the plant, which was acquired with thermal imaging In addition, other systems have been developed to schedule irrigation of crops and optimize water use by means of a crop water stress index (CWSI) The empirical CWSI was first defined over 30 years ago This index was later calculated

using measurements of infrared canopy temperatures, ambient air temperatures, and atmospheric vapor pressure deficit values to determine when to irrigate broccoli using drip irrigation Irrigation systems can also be automated through information on volumetric water content of soil, using dielectric moisture sensors to control actuators and save water, instead of a pre-determined irrigation schedule at a particular time of the day and with a specific duration. An irrigation controller is used to open a solenoid valve and apply watering to bedding plants (impatiens, petunia, salvia, and vinca) when the volumetric water content of the substrate drops below a set point

Other authors have reported the use of remote canopy tem-perature to automate cotton crop irrigation using infrared ther-mometers. Through a timed temperature threshold, automatic irrigation was triggered once canopy temperatures exceeded the threshold for certain time accumulated per day. Automatic irrigation scheduling consistently has shown to be valuable in optimizing cotton yields and water use efficiency with respect to manual irrigation based on direct soil water mea-surements .

An alternative parameter to determine crop irrigation needs is estimating plant evapotranspiration (ET). ET is affected by weather parameters, including solar radiation, temperature, relative humidity, wind speed, and crop factors, such as stage of growth, variety and plant density, management elements, soil properties, pest, and disease control Systems based on ET have been developed that allow water savings of up to $42 \%$ on timebased irrigation schedule In Florida, automated switching tensiometers have been used in combination with ET calculated from historic weather data to control automatic irrigation schemes for papaya plants instead of using fixed scheduled ones. Soil water status and 
ET-based irrigation methods resulted in more sustainable practices compared with set schedule irrigation because of the lower water volumes applied.

An electromagnetic sensor to measure soil moisture was the basis for developing an irrigation system at a savings of

$53 \%$ of water compared with irrigation by sprinklers in an area of $1000 \mathrm{~m}^{2}$ of pasture.substrate moisture content .machine converted to make sprinkler nozzles controllable. The network consisted of five sensing stations and a weather station. Each of the sensing stations contained a data logger with two soil water reflectometers, a soil temperature sensor, and Bluetooth communication. Using the network information and the irrigation machine positions through a differential GPS, the software controlled the sprinkler with application of the appropriate amount of water. Software dedicated to sprinkler control has been variously discussed.

A data acquisition system was deployed for monitoring crop conditions by means of soil moisture and soil, air, and canopy temperature measurement in cropped fields. Data were downloaded using a handheld computer connected via a serial port for analysis and storage. Another system used to achieve the effectiveness of water management was developed based on a WSN and a weather station for Internet monitoring of drainage water using distributed passive capillary wicktype lysimeters. Water flux leached below the root zone under an irrigated cropping system was measured .There are hybrid architectures, wireless modules are located inside the green-house where great flexibility is required, and wired modules are used in the outside area as actuator controllers.

The development of WSNs based on microcontrollers and communication technologies can improve the current meth-ods of monitoring to support the response appropriately in real time for a wide range of applications, considering the requirements of the deployed area, such as terrestrial, underground, underwater, multimedia, and mobile. These applications involve military operations in scenarios of bat-tlefield, urban combat, and force protection, with tasks of presence, intrusion, ranging, imaging, detection of chemical, toxic material, biological, radiological, nuclear, and explo-sive. In addition, sensor networks have been used in health care purposes for monitoring, alerting, assistance, and actuating with security and privacy to support real-time data transmission. Vital sign monitoring, such as ECG, heart rate, body temperature, has been integrated in hospitals and homes through wearable or e-textile providing reports and alerts to personal in case of emergency and tracking the location of patients within the hospital limits. WSNs have been used to remote monitor healthcare of dependent people at their homes through several biomedical sensors such as ECG, blood pressure, body temperature, and body motion. Home applications comprised wireless embedded sensors and actuators that enable monitoring and control. For comfort and efficient energy management, household devices have been controlled through sensors that monitor parameters such as temperature, humidity, light, and presence, avoiding waste of energy. Sensor networks have been used for security pur-poses, based on several sensors such as smoke detectors, gas sensors, and motion sensors, to detect possible risk situations that trigger appropriate actions in response, such as send an alert to a remote center through wireless communication. In industrial environments, WSNs have been installed to provide real-time data acquisition for inventory management, to equipment monitoring for control with appropriate actions, reducing human errors and preventing manufacturing down-time. For example, industrial WSN have been imple-mented to motor fault diagnosis and for the monitoring of the temperaturesensitive products during their distribution has been proposed. In addition, there are wireless systems for structural identification under environmental an operationalparameters, such as load in bridges.

In environmental applications, sensor networks have been used to monitor a variety of environmental parameters or conditions in marine, soil, and atmospheric contexts. Environmental parameters, including humidity, pressure, tem-perature, soil water content, and radiation with different spatial and temporal resolution and for event detection such as disaster monitoring, pollution conditions, floods, forest fire, and debris flow is continuously monitored. Applications in agriculture have been used to provide data for appropriate management, such as monitoring of environmental condi-tions like weather, soil moisture content, soil temperature, soil fertility, mineral content, and weed disease detection, monitoring leaf temperature, moisture content, and monitoring growth of the crop, automated irrigation facility and storage of agricultural products.

Various commercial WSNs exist, ranging from limited and low-resolution devices with sensors and embedded proces-sors to complete and expensive acquisition systems that sup-port diverse sensors and include several communication fea-tures. Recent advances in microelectronics and wireless technologies created low-cost and low-power components, which are important issues especially for such systems such as WSN. Power management has been addressed in both hardware and software with new electronic designs and operation techniques. The selection of a microprocessor becomes important in power aware design. Modern CMOS and micro-electro-mechanical systems (MEMS) technologies allowed manufacturers to produce on average every three years a enhance generation of circuits by integrating sensors, signal conditioning, signal processing, digital output options, com-munications, and power supply units. For example, the parallel combination of a battery and a supercapacitor has been used to extend the runtime of low-power wireless sensor nodes.

Energy harvesting mechanisms have been employed, 
in cases where it is difficult for changing or recharging batteries, hence this strategy has involved combining it with efficient power management algorithms to optimize battery lifetime. Power harvesting is a complementary approach that depends on ambient energy sources, including environmental vibration, human power, thermal, solar, and wind that can be converted into useable electrical energy. On the other hand, several strategies have been implemented to reduce power con-sumption, such as power-aware protocols, resource and task management, communication, topology control and routing, models based on events, and congestion control mechanism to balance the load, prevent packet drops, and avoid network deadlock using a combination of predeployed group keys that allow the dynamic creation of high security subnetworks and optimizes energy efficiency of sensor networks. For instance, energy-saving strategies have been achieved through scheduling, sleep or wake up schemes, and adaptive radio frequency (RF) in nodes, and choosing a network configuration. There are also algorithms to maximize the network coverage ratio with a predefined balance the energy consumption in the whole WSN, to reduce both the transmission and the computational loads at the node level, and to estimate online the optimal sampling frequencies for sensors.

In a wireless node, the radio modem is the major power consuming component; recently, wireless standards have been established with medium access control protocols to provide multitask support, data delivery, and energy efficiency per-formance, such as the standards for wireless local area network, IEEE $802.11 \mathrm{~b}$ (WiFi) and wireless personal area network (WPAN), IEEE 802.15.1 (Bluetooth), IEEE 802.15.3 (UWB) [59], and IEEE 802.15.4 (ZigBee), and those open wireless communication standards for Internet pro-tocol version 6 (IPv6) over low-power wireless personal area networks 6LoWPAN , wireless highway addressable remote transducer WirelessHART, and ISA100.11a [64] developed by the International Society of Automation.

In this paper, the development of the deployment of an automated irrigation system based on microcontrollers and wireless communication at experimental scale within rural areas is presented. The aim of the implementation was to demonstrate that the automatic irrigation can be used to reduce water use. The implementation is a photovoltaic powered automated irrigation system that consists of a distributed wireless network of soil moisture and temperature sensors deployed in plant root zones. Each sensor node involved a soil-moisture probe, a temperature probe, a microcontroller for data acquisition, and a radio transceiver; the sensor mea-surements are transmitted to a microcontroller-based receiver. This gateway permits the automated activation of irrigation when the threshold values of soil moisture and temperature are reached. Communication between the sensor nodes and the data receiver is via the Zigbee protocol under the IEEE
802.15.4 WPAN. This receiver unit also has a duplex communication link based on a cellular-Internet interface, using general packet radio service (GPRS) protocol, which is a packet-oriented mobile data service used in $2 \mathrm{G}$ and $3 \mathrm{G}$ cellular global system for mobile communications (GSM). The Internet connection allows the data inspection in real time on a website, where the soilmoisture and temperature levels are graphically displayed through an application interface and stored in a database server. This access also enables direct programming of scheduled irrigation schemes and trigger values in the receiver according the crop growth and season management. Because of its energy autonomy and low cost, the system has potential use for organic crops, which are mainly located in geographically isolated areas where the energy grid is far away.

\section{Automated Irrigation SyStem}

The automated irrigation system hereby reported, consisted of two components, wireless sensor units (WSUs) and a wireless information unit (WIU), linked by radio transceivers that allowed the transfer of soil moisture and temperature data, implementing a WSN that uses ZigBee technology. The WIU has also a GPRS module to transmit the data to a web server via the public mobile network. The information can be remotely monitored online through a graphical application through Internet access devices.

\section{A. Wireless Sensor Unit}

A WSU is comprised of a RF transceiver, sensors, a microcontroller, and power sources. Several WSUs can be deployed in-field to configure a distributed sensor network for the automated irrigation system. Each unit is based on the microcontroller PIC24FJ64GB004 (Microchip Technolo-gies, Chandler, AZ) that controls the radio modem XBee Pro S2 (Digi International, Eden Prairie, MN) and processes information from the soil-moisture sensor VH400 (Vegetronix, Sandy, UT), and the temperature sensor DS1822 (Maxim Integrated, San Jose, CA). These components are powered by rechargeable AA 2000mAh Ni-MH CycleEnergy batteries (SONY, Australia). The charge is maintained by a photovoltaic panel MPT4.8-75 (PowerFilm Solar, Ames, IN) to achieve full energy autonomy. The microcontroller, radio modem, rechargeable batteries, and electronic components were encap-sulated in a waterproof Polyvinyl chloride (PVC) container (Fig. 2). These components were selected to minimize the power consumption for the proposed application.

1) Single-Chip PIC24FJ64GB004: A 16-bit microcontroller with 44-pins and nanoWatt XLP technology that operates in a range 2.0 to $3.6 \mathrm{~V}$ at 8 $\mathrm{MHz}$ with internal oscillator. It has up to 25 digital input/output ports, 13-, 10-bit analog-to-digital converters 
(ADC), two serial peripheral interface modules, two - stop bit, 8 - data bits.

I2C, two UART, 5 16-bit timers, $64 \mathrm{~KB}$ of program The WSUs were configured such as end devices to memory, $8 \mathrm{~KB}$ of SRAM, and hardware real-time deploy a networking topology point-to-point based on a clock/calendar (RTCC). The microcontroller is well suited coordinator that was implemented by the XBee radio for this remote application, because of its low-power modem of the WIU. An end device has the following operating current, which is $175 \mu \mathrm{A}$ at $2.5 \mathrm{~V}$ at $8 \mathrm{MHz}$ characteristics: 1) it must join a ZigBee PAN before it can and $0.5 \mu \mathrm{A}$ for standby current in sleep mode including transmit or receive data; 2) cannot allow devices to join the RTCC.

the network; 3) must always transmit and receive RF data The microcontroller was programmed in $\mathrm{C}$ compiler through its parent; 4) cannot route data; and 5) can enter 4.12 (Custom Computer Services, Waukesha, WI) with low power modes to conserve power and can be battery the appro-priate algorithm (Fig. 3) for monitoring the soil- powered. The least significant byte of the unique 64-bit moisture probe through an analog-to-digital port and address is used to label the information of the soil the soil-temperature probe through another digital port, moisture and temperature for each WSU in the implemented in 1-Wire communication protocol. A network. This byte is registered in the WIU as the battery voltage monitor is included through a high- identifier (ID) associated to each WSU. As shown in the impedance voltage divider coupled to an analog-to- sample frames to request date/time, receive date/time, digital port. The data are packed with the cor- and send data packaged to the WIU (Fig. 4).

responding identifier, date, and time to be transmitted 3) Soil Sensor Array: The sensor array consists of two via XBee radio modem using a RS-232 protocol soil sensors, including moisture and temperature that are through two digital ports configured as transmitter (TX) inserted in

and receiver (RX), respectively. After sending data, the the root zone of the plants. The VH400 probe was microcontroller is set in sleep mode for certain period selected to estimate the soil moisture because of low according to the sensor sampling rate desired, whereas power consumption $(<7 \mathrm{~mA})$ and low cost. The probe the internal RTCC is running. This operation mode measures the dielectric constant of the soil using allows energy savings. When the WSU is launched for transmission line techniques at $80 \mathrm{MHz}$, which is first time, the algorithm also inquires the WIU, the date and time to program the RTCC, and periodically updates it for synchronization.

2) ZigBee Modules: ZigBee (over IEEE 802.15.4) technol-ogy is based on short range WSN and it was selected for this battery-operated sensor network because of its low cost, low power consumption, and greater useful range in comparison with other wireless technologies like Bluetooth (over IEEE 802.15.1), UWB (over IEEE 802.15.3), and Wi-Fi (over IEEE 802.11) [67]. The ZigBee devices operate in industrial, scien-tific, and medical $2.4-\mathrm{GHz}$ radio band and allow the operation in a so-called mesh networking architecture, which can be differentiated into three categories: 1) coordinator; 2) router; and 3) end device. From a wide range of commercial ZigBee devices, the XBee-PRO S2 is an appropriate original equipment manufac-turer module to establish communication between a WSU and the WIU because of its long-range operation and reliability of the sensor networking architecture. The XBee-PRO S2 is a RF modem with integrated chip antenna, 20-pins, and 13 general purpose input/output (GPIO) ports available of which four are ADC. It can operate up to a distance of $1500 \mathrm{~m}$ in outdoor line-of-sight with $170 \mathrm{~mA}$ of TX peak current and $45 \mathrm{~mA}$ for $\mathrm{RX}$ current at $3.3 \mathrm{~V}$ and power-down current of $3.5 \mu \mathrm{A}$.

The XBee radio modem of each WSU is powered at 3.3 $\mathrm{V}$ through a voltage regulator ADP122AUJZ-3.3-R7 (Analog Devices, Norwood, MA) and interfaced to the host microcon-troller through its serial port, a logic-level asynchronous serial, and voltage compatible UART configured at 9600 baud rate, no - parity, 1 - start bit, 1 insensitive to water salinity, and provides an output range between 0 and $3.0 \mathrm{~V}$, which is proportional to the volumetric water content (VWC) according to a calibration curve provided by the manufacturer. The sensor was powered at $3.3 \mathrm{~V}$ and monitored by the microcontroller through an ADC port.Soil temperature measurements were made through the dig-ital thermometer DS1822. The sensor converts temperature to a 12-bit digital word and is stored in 2-B temperature registers, corresponding to increments of $0.0625{ }^{\circ} \mathrm{C}$. The temperature is required through a reading command and transmitted using 1Wire bus protocol implemented in the microcontroller

through one digital port. The thermometer has \pm 2.0 ${ }^{\circ} \mathrm{C}$ accuracy over $-10{ }^{\circ} \mathrm{C}$ to $+85{ }^{\circ} \mathrm{C}$ temperature range and a unique 64-bit serial number. The sensor is a 3-pin single-chip and TO92 package that was embedded in a metal capsule and sealed in a waterproof PVC cylindrical container.

To calibrate the soil moisture, several samples were prepared with $1 \mathrm{~kg}$ of dry soil from the crop area. Its composition was loamy sand with $80 \%$ sand separate, $4.5 \%$ clay separate, and $15.6 \%$ silt separate. The soil water holding capacity was of $20.7 \%$ VWC corresponding to measured output voltages of $1.45 \mathrm{~V}$. The temperature sensors were calibrated through a reference mercury thermometer CT40, with $0.1{ }^{\circ} \mathrm{C}$ divisions and a range from $-1{ }^{\circ} \mathrm{C}$ to $51{ }^{\circ} \mathrm{C}$. The thermometer and the temperature sensors were placed in an insulated flask filled with mineral oil at $10{ }^{\circ} \mathrm{C}$ and 40 ${ }^{\circ} \mathrm{C}$.

4) Photovoltaic Cell: To maintain the charge of the WSU batteries, a solar panel MPT4.8-75 was employed. Each solar panel delivers $50 \mathrm{~mA}$ at $4.8 \mathrm{~V}$, which is sufficient energy to maintain the voltage of the three 
rechargeable batteries. A MSS1P2U Schottky diode When the server receives a request for the web page, (Vishay, Shelton, CT) is used to prevent the solar it inserts each data to the corresponding field in the module and to drain the battery when is in the dark. database.

The solar panel is encapsulated in a 3-mm clear This link is bidirectional and permits to change the polyester film with dimensions of $94 \mathrm{~mm} \times 75 \mathrm{~mm}$. threshold values through the website interface; scheduled This flexible panel was mounted on a PVC prismatic watering or remote watering can be performed.

base $(100 \mathrm{~mm} \times 80 \mathrm{~mm} \times 3.17 \mathrm{~mm})$ that is fastened in The WIU has also a push button to perform manual the upper part of a PVC pole allowing for the correct irriga-tion for a programmed period and a LED to indicate alignment of the photovoltaic panel to the sun. The stick when the information package is received. All the WIU is $50 \mathrm{~cm}$ of length and $12.5 \mathrm{~mm}$ of diameter; the lower processes can be monitored through the RS-232 port. end of the pole had a tip end to be buried.

\section{B. Wireless Information Unit}

The WIU includes a function that synchronizes the WSUs at noon for monitoring the status of each WSU. are received, identified, recorded, and analyzed in the Besides this action, an email is sent to alert the system WIU. The WIU consists of a master microcontroller administrator. 2) GPRS Module: The MTSMC-G2-SP is a PIC24FJ64GB004, an XBee radio modem, a GPRS cellular modem embedded in a 64-pins universal socket module MTSMC-G2-SP (MultiTech Systems, Mounds that offers standards-based quad-band GSM/GPRS Class View, MN), an RS-232 inter-face MAX3235E (Maxim 10 performance. This GPRS modem includes an Integrated, San Jose, CA), two electronic relays, two embedded transmission control protocol/Internet protocol $12 \mathrm{~V}$ dc $1100 \mathrm{GPH}$ Livewell pumps (Rule-Industries, stack to bring Internet connectivity, a UFL antenna Gloucester, MA) for driving the water of the tanks, and a connector and subscriber identity module (SIM) socket. deep cycle $12 \mathrm{~V}$ at 100 -Ah rechargeable battery $\mathrm{L}$ - The module is capable of transfer speeds up to 115.2 24M/DC-140 (LTH, Mexico), which is recharged by a $\mathrm{K} \mathrm{b} / \mathrm{s}$ and can be interfaced directly to a UART or solar panel KC130TM of $12 \mathrm{~V}$ at $130 \mathrm{~W}$ (Kyocera, microcontroller using AT commands. It also includes an Scottsdale, AZ) through a PWM charge controller SCI-120 onboard LED to display network status.

(Syscom, Mexico). All the WIU electronic components were encapsulated in a waterproof PVC box as shown in Figs. 5 and 6 . The WIU can be located up to 1500 -m lineof-sight from the WSUs placed in the field.

1) Master Microcontroller: The functionality of the WIU is based on the microcontroller, which is programmed to perform diverse tasks, as is shown in Fig. 7. The first task of the program is to download from a web server the date and time through the GPRS module. The WIU is ready to transmit via XBee the date and time for each WSU once powered. Then, the microcontroller receives the information package transmitted by each WSU that conform the WSN.

These data are processed by the algorithm that first identifies the least significant byte of a unique 64-bit address encap-sulated in the package received. Second, the soil moisture and temperature data are compared with programmed values of minimum soil moisture and maximum soil temperature to activate the irrigation pumps for a desired period. Third, the algorithm also records a $\log$ file with the data in a solid state memory 24FC1025 (Microchip Technologies, Chandler, AZ) with a capacity of $128 \mathrm{kB}$. Each $\log$ is 12-B long, including soil moisture and temperature, the battery voltage, the WSU ID, the date, and time generated by the internal RTCC. If irrigation is provided, the program also stores a register with the duration of irrigation, the date, and time. Finally, these data and a greenhouse ID are also transmitted at each predefined time to a web server through HTTP via the GPRS module to be deployed on the Internet web application in real time.

.3) Watering Module: The irrigation is performed by con-trolling the two pumps through 40-A electromagnetic relays connected with the microcontroller via two optical isolators CPC1004N (Clare, Beverly. MA). The pumps have a power consumption of $48 \mathrm{~W}$ each and were fed by a 5000-1 water tank.

Four different irrigation actions (IA) are implemented in the WIU algorithm:

1) fixed duration for manual irrigation with the push button; 2) scheduled date and time irrigations through the web page

for any desired time;

3) automated irrigation with a fixed duration, if at least one soil moisture sensor value of the WSN drops below the programmed threshold level;

4) automated irrigation with a fixed duration, if at least one soil temperature sensor value of the WSN exceeds the programmed threshold level.

\section{Web Application}

Graphical user interface software was developed for real-time monitoring and programming of irrigation based on soil moisture and temperature data. The software application permits the user to visualize graphically the data from each WSU online using any device with Internet

Besides the soil-moisture and temperature graphs, the web application displays the total water consumption and the kind of the IA.

The web application also enabled the user direct program-ming of scheduled irrigation schemes and 
adjusting the trigger values in the WIU according to the crop species and season management. All the information is stored in a database. The web application for monitoring and programming was coded in $\mathrm{C \#}$ language of Microsoft Visual Studio 2010. The database was implemented in SQL Server 2005.

III. IRRIGATION SYSTEM OPERATION

The system was tested in a $2400-\mathrm{m}^{2}$ greenhouse, located near San Jose del Cabo, Baja California Sur (BCS), Mexico (23 $10.841^{\prime} \mathrm{N}, 109^{\circ} 43.630^{\prime} \mathrm{W}$ ) for organic sage (Salvia officinalis) production. The greenhouse had 56 production beds covered with plastic. Each bed was 14-m long and ssssin the roo zone of the plants. These three units allowed data redundancy to ensure irrigation control. The algorithm considered the values from the WSU-54, 55, and 56, if one reached the threshold values the automated irrigation was performed.

The pumping rate provided $10 \mathrm{ml} / \mathrm{min} /$ drip hole, which was measured in the automated irrigation zone in six different drip holes.

In accordance with the organic producer's experience, a minimum value of $5 \%$ VWC for the soil was established as the moisture threshold level and $30{ }^{\circ} \mathrm{C}$ as the temperature threshold level for the automated irrigation modes (IA-3 and IA-4, respectively). Initially, the scheduled irrigation (IA-2) of $35 \mathrm{~min} /$ week was used during the first six weeks. After that, the scheduled irrigation was set at $35 \mathrm{~min}$ three times per week. Sage cultivation finalized after 136 days.

During the cultivation, several automated irrigation periods were carried out by the system because of the soil-moisture (IA-3) or temperature (IA-4) levels, regardless of the scheduled irrigation (IA-2). All data were uploaded each hour to the web server for remote supervision. For instance, data of five days are shown (Fig. 10). The first graph shows soil temperatures. The vertical bars indicate automated irrigation periods triggered by temperature when soil temperature was above the threshold value $\left(30{ }^{\circ} \mathrm{C}\right)$. The second graph shows

(curve a) was lower than the automated irrigation (curve b). The daily mean VWC for the traditional irrigation practice (curve c) was almost constant $>16 \%$, whereas that for the automated irrigation (curve d) was below $10 \%$. In addition, the accumulated water used are shown corresponding to 14 beds

for each irrigation system. The total water requirement was $341 \mathrm{~m}^{3}$ for the traditional one and $29 \mathrm{~m}^{3}$ for the automated

one. Then, the automated irrigation used $\sim 90 \%$ less water with respect to the traditional irrigation practice.

Another three automated irrigation systems have been tested along 18 months in other places in BCS, Mexico: El Pescadero (23 $\left.21.866^{\prime} \mathrm{N}, 110^{\circ} 10.099^{\prime} \mathrm{W}\right)$, El Comitan-CIBNOR (24 $\left.7.933^{\prime} \mathrm{N}, 110^{\circ} 25.416^{\prime} \mathrm{W}\right)$, and Los Ara-dos (24 47.1' N, $111^{\circ} 11.133^{\prime}$ W). In these three places, programmed irrigations (IA-2) were compared with trig-gered irrigations (IA-3 and IA-4), water savings $\sim 60 \%$ were obtained.

For cases such as Los Arados, it was found that the signal receiving strength was too low and the Internet connection could not be established, hence in this case all data were stored into the solid state memory of the WIU.

Power consumption of a WSU was measured through current oscilloscope (UNI-T UT81B) in the monitoring and sleep operational modes. Each hour, the soil-moisture temperature data were transmitted to the WIU. Before transmitting the data, the XBee of the WSU was powered on through the voltage regulator that was enabled for a period of $20 \mathrm{~s}$ by the microcontroller, which was a long enough time for the radio modem to wake up and transmit the data. Then, the total average power consumption was kept at $0.455 \mathrm{mAh}$. The charge-discharge cycle of the batteries is shown for 20 days in the winter with the solar panel connected and disconnected using the data registered by the battery voltage monitor. The solar radiation for those days is shown in . Thus, the photovoltaic panel and the batteries provide sufficient energy to maintain the WSU running for the whole crop season at almost any latitude, due the low energy

However, the total average power consumption was $\sim 4$ Ah per day considering the two irrigation pumps.

The automated irrigation system implemented is a cost-effective alternative for agriculture; the cost of each WSU is $\sim 100$ U.S. dollars (Table I), and the WIU cost is $\sim 1800$ U.S. dollars

\section{Conclusion}

The automated irrigation system implemented was found to be feasible and cost effective for optimizing water resources for agricultural production. This irrigation system allows cultivation in places with water scarcity thereby improving sustainability.

The automated irrigation system developed proves that the use of water can be diminished for a given amount of fresh biomass production. The use of solar power in this irrigation system is pertinent and significantly important for organic crops and other agricultural products that are geographically isolated, where the investment in electric power supply would be expensive. The irrigation system can be adjusted to a variety of specific crop needs and requires minimum maintenance. The modular configuration of the automated irrigation system allows it to be scaled up for larger greenhouses or open fields. In addition, other applications such as temperature monitoring in compost production can be easily implemented. The Internet controlled duplex communication system provides a powerful decisionmaking device concept for adaptation to several cultivation scenarios. Furthermore, the Internet link allows the supervision through mobile telecommunication devices, such as a smart-phone.

Besides the monetary savings in water use, the importance of the preservation of this natural resource 
justify the use of this kind of irrigation system.

\section{REFERENCES}

[1] W. A. Jury and H. J. Vaux, "The emerging global water crisis: Managing scarcity and conflict between water users," $A d v$. Agronomy, vol. 95, pp. 1-76, Sep. 2007.

[2] X. Wang, W. Yang, A. Wheaton, N. Cooley, and B. Moran, "Efficient registration of optical and IR images for automatic plant water stress assessment," Comput. Electron. Agricult., vol. 74, no. 2, pp. 230-237, Nov. 2010.

[3] G. Yuan, Y. Luo, X. Sun, and D. Tang, "Evaluation of a crop water stress index for detecting water stress in winter wheat in the North China Plain," Agricult. Water Manag., vol. 64, no. 1, pp. 29-40, Jan. 2004

[4] S. B. Idso, R. D. Jackson, P. J. Pinter, Jr., R. J. Reginato, and J. L. Hatfield, "Normalizing the stress-degreeday parameter for environ-mental variability," Agricult. Meteorol., vol. 24, pp. 45-55, Jan. 1981.

[5] Y. Erdem, L. Arin, T. Erdem, S. Polat, M. Deveci, H. Okursoy, and H. T. Gültas, "Crop water stress index for assessing irrigation scheduling of drip irrigated broccoli (Brassica oleracea L. var. italica)," Agricult. Water Manag., vol. 98 , no. 1 , pp. 148-156, Dec. 2010.

[6] K. S. Nemali and M. W. Van Iersel, "An automated system for con-trolling drought stress and irrigation in potted plants," Sci. Horticult., vol. 110, no. 3, pp. 292-297, Nov. 2006.

[7] S. A. O'Shaughnessy and S. R. Evett, "Canopy temperature based sys-tem effectively schedules and controls center pivot irrigation of cotton," Agricult. Water Manag., vol. 97, no. 9, pp. 1310-1316, Apr. 2010.

[8] R. G. Allen, L. S. Pereira, D. Raes, and M. Smith, Crop Evapotranspiration-Guidelines forComputing Crop Water Requirements-FAO Irrigation and Drainage Paper 56. Rome, Italy: FAO, 1998.

[9] S. L. Davis and M. D. Dukes, "Irrigation scheduling performance by evapotranspiration-based controllers," Agricult. Water Manag., vol. 98, no. 1, pp. 19-28, Dec. 2010.

[10] K. W. Migliaccio, B. Schaffer, J. H. Crane, and F. S. Davies, "Plant response to evapotranspiration and soil water sensor irrigation schedul-ing methods for papaya production in south Florida," Agricult. Water Manag., vol. 97, no. 10, pp. 1452-1460, Oct. 2010.

[11] J. M. Blonquist, Jr., S. B. Jones, and D. A. Robinson, "Precise irrigation scheduling for turfgrass using a subsurface electromagnetic soil moisture sensor," Agricult. Water Manag., vol. 84 , nos. $1-2$, pp. 153-165, Jul. 2006

[12] O. M. Grant, M. J. Davies, H. Longbottom, and C. J. Atkinson, "Irrigation scheduling and irrigation systems: Optimising irrigation effi-ciency for container ornamental shrubs," Irrigation Sci., vol. 27, no. 2, pp. 139-153, Jan. 2009.

[13] Y. Kim, R. G. Evans, and W. M. Iversen, "Remote sensing and control of an irrigation system using a distributed wireless sensor network," IEEE Trans. Instrum. Meas., vol. 57, no. 7, pp. 1379-1387, Jul. 2008

[14] Y. Kim and R. G. Evans, "Software design for wireless sensor-based site-specific irrigation," Comput. Electron. Agricult., vol. 66, no. 2, pp. 159-165, May 2009.

[15] D. K. Fisher and H. A. Kebede, "A low-cost microcontroller-based system to monitor crop temperature and water status," Comput. Electron. Agricult., vol. 74, no. 1, pp. 168-173, Oct. 2010.

[16] Y. Kim, J. D. Jabro, and R. G. Evans, "Wireless lysimeters for real-time online soil water monitoring," Irrigation Sci., vol. 29, no. 5, pp. 423-430, Sep. 2011.

[17] O. Mirabella and M. Brischetto, "A hybrid wired/wireless networking infrastructure for greenhouse management," IEEE Trans. Instrum. Meas., vol. 60, no. 2, pp. 398-407, Feb. 2011.

[18] I. F. Akyildiz, W. Su, Y. Sankarasubramaniam, and E. Cayirci,
"A survey on sensor networks," IEEE Commun. Mag., vol. 40, no. 8, pp. 104-112, Aug. 2002.

[19] J. Yick, B. Mukherjee, and D. Ghosal, "Wireless sensor network survey," Comput. Netw., vol. 52, no. 12, pp. 22922330, Aug. 2008.

[20] M. Winkler, K.-D. Tuchs, K. Hughes, and G. Barclay, "Theoretical and practical aspects of military wireless sensor networks," J. Telecommun. Inf. Technol., vol. 2, pp. 37-45, Apr./Jun. 2008.

[21] M. P. Durisic, Z. Tafa, G. Dimic, and V. Milutinovic, "A survey of military applications of wireless sensor networks," in Proc. MECO, Jun. 2012, pp. 196-199.

[22] M. C. Rodríguez-Sánchez, S. Borromeo, and J. A. HernándezTamames, "Wireless sensor networks for conservation and monitoring cultural assets," IEEE Sensors J., vol. 11, no. 6, pp. 1382-1389, Jun. 2011.

[23] G. López, V. Custodio, and J. I. Moreno, "LOBIN: E-textile and wireless-sensor-network-based platform for healthcare monitoring in future hospital environments," IEEE Trans. Inf. Technol. Biomed., vol. 14, no. 6, pp. 1446-1458, Nov. 2010

[24] J. M. Corchado, J. Bajo, D. I. Tapia, and A. Abraham, "Using heterogeneous wireless sensor networks in a telemonitoring system for healthcare," IEEE Trans. Inf. Technol. Biomed., vol. 14, no. 2, pp. 234-240, Mar. 2010

[25] G. X. Lee, K. S. Low, and T. Taher, "Unrestrained measurement of arm motion based on a wearable wireless sensor network," IEEE Trans. Instrum. Meas., vol. 59, no. 5, pp. 1309 1317, May 2010.

[26] D.-M. Han and J.-H. Lim, "Smart home energy management system using IEEE 802.15.4 and ZigBee," IEEE Trans. Consum. Electron., vol. 56, no. 3, pp. 1403-1410, Aug. 2010

[27] C. Gomez and J. Paradells, "Wireless home automation networks: A survey of architectures and technologies," IEEE Commun. Mag., vol. 48, no. 6, pp. 92-101, Jun. 2010

[28] M. Bertocco, G. Gamba, A. Sona, and S. Vitturi, "Experimental charac-terization of wireless sensor networks for industrial applications," IEEE Trans. Instrum. Meas., vol. 57, no. 8, pp. 1537-1546, Aug. 2008

[29] V. C. Gungor and G. P. Hancke, "Industrial wireless sensor networks: Challenges, design principles, and technical approaches," IEEE Trans. Ind. Electron., vol. 56, no. 10, pp. 4258-4265, Oct. 2009.

[30] L. Hou and N. W. Bergmann, "Novel industrial wireless senso networks for machine condition monitoring and fault diagnosis," IEEE Trans. Instrum. Meas., vol. 61, no. 10, pp. 2787-2798, Oct. 